\title{
Directional Growth of Optic Nerve Axons and Processive Gliogenesis
}

Steven L. Bernstein ${ }^{1}$, Yan Guo ${ }^{1}$, and Zara Mehrabian ${ }^{1}$

1. Department of Ophthalmology and Visual Sciences, University of Maryland School of Medicine, Baltimore, USA.

The axons of retinal ganglion cells (RGCs) comprise the optic nerve (ON)[1]. The postnatal ON in humans and rodents grows in length by over $80 \%$ [2]. However, the mechanism of postnatal axonal growth is still poorly understood, and has been hypothesized by some to occur diffusely, since axon myelination has already occurred. We recently determined that the most anterior portion of the $\mathrm{ON}$ in mammals (the optic nerve lamina region, ONLR) contains a SOX2(+) neural progenitor cell (NPC) niche that can ultimately give rise to oligodendrocyte progenitors (OPCs), and all major glial forms: Type 1 and 2 astrocytes and oligodendrocytes. We wanted to determine the role of this SOX2(+)ONLR niche during the period of postnatal axonal growth. We used animals whose SOX2(+) cells would self-delete using a genetic construct that expresses Diphtheria toxin-Subunit A (DTA). Even a single molecule of DTA results in cell death within three days.

Transgenic mice constructed with the SOX2-ER2-Cre cassette were mated with homologousrecombined ROSA26-LoxP-(DTA) mutant mice, to create a double heterozygote mutant that expresses diphtheria toxin-A (DTA) in SOX2-expressing cells upon treatment with tamoxifen (TAM) or its active metabolite, 4-hydroxytamoxifen (4OHT). Animals were genotyped, and double heterozygotes (usually $\sim 50 \%$ of litters) were treated at $19 \mathrm{dPN}$ with $4 \mathrm{OHT}$ using unilateral retrobulbar (Local: behind the eye) injection. Control animals received vehicle alone (Kolliphor). We utilized local administration since systemic administration of TAM or 4OHT was found to be fatal within one week to double heterozygotes, presumably due to the requirement for $\mathrm{SOX} 2(+)$ expression in bone marrowmyelogenous progenitors (Human Protein Atlas: https://www.proteinatlas.org/ ENSG00000181449SOX2/tissue/bone+marrow). Animals were euthanized by perfusion 14d later (PN d33), which corresponds to near the end of ON growth. ONs were either prepared for immunohistochemistry (4\% paraformaldehyde), or for ultrastructure (buffered glutaraldehyde-paraformaldehyde). Both anterior ON region (1.5-2mm from the globe) and mid-ON region ( $5 \mathrm{~mm}$ from the globe) were evaluated. Tissues for ultrastructure were shadowed with uranyl acetate, embedded in Epon, and cross-sectioned at 200nm. We also evaluated RGC loss using immunostaining for Brn3a; an RGC-expressed nuclear transcription factor, and performing stereology.

The ONs of animals who received vehicle had a typical distribution of tightly packed large- and small axons $1.5 \mathrm{~mm}$ from the globe (Fig 1). These axons were normally myelinated, with few unmyelinated axons apparent. In contrast, ONs from animals treated with 4OHT revealed a loss of axons, with reduced packing density, reduced myelination (reduced G-ratio) and many unmyelinated axons. Axons examined at mid-region were normally myelinated. 4OHT treated eyes had reduced numbers of RGCs, which were seen to be lost in a patchy fashion. Immunostaining revealed a loss of SOX2(+) cells and reduced nestin immunopositivity, consistent with loss of NPCs.

Our results suggest that during the postnatal period, the myelinated RGC axon grows from the unmyelinated anterior end closest to the axon hillock, rather than by diffuse expansion, and that ONLRNPCs are responsible for processive gliogenesis: that is, the ONLR-NPCs generate the glia cells used to myelinate the growing nerve in a unilateral fashion, and are responsible for giving rise to all macroglial cell components in the growing ON. Loss of ONLR-NPCs during the active growth period of the otic nerve results in RGC loss and optic nerve hypomyelination, with resultant hypoplasia. 


\section{References:}

[1] Miller, N. in "Anatomy and Physiology of Optic Nerve", in Walsh and Hoyts Clinical Neuro Ophthalmology, ed.N.R. Miller, (Williams and Wilkins, Baltimore), p. 41.

[2] S. Bernstein et al, Eye 30 (2016), p.1378.
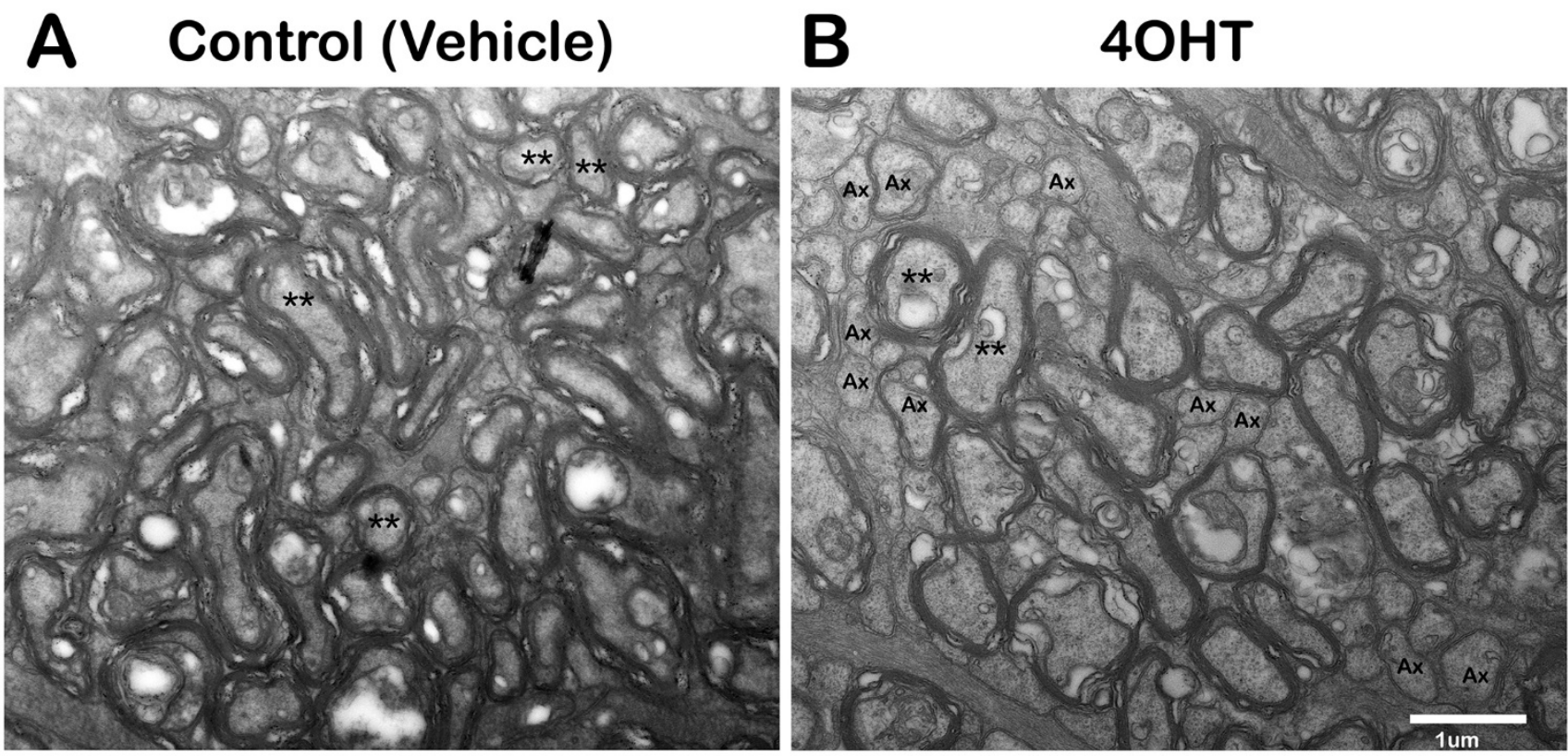

Figure 1. Ultrastructural analysis of the RGC axons in the anterior ON of a $33 \mathrm{~d}$ SOX2-ER2-Cre $\mathrm{X}$ ROSA26-td-Tomato-LoxP(GFP) double mutant two weeks post-local administration of: A. vehicle (Kolliphor). B. 4-Hydroxytamoxifen (4OHT). Axons in the $\mathrm{ON}$ of the vehicle treated eye are uniformly and heavily myelinated (double asterisks), with a mixture of large and small diameter axons. The 4OHT treated $\mathrm{ON}$ sectioned at a similar distance from the eye shows a mixture of myelinated and unmyelinated- and partially myelinated axons ( $\mathrm{Ax})$, with the myelinated axons exhibiting less heavy myelination throughout (hypomyelination). There is less axonal density overall. Scale bar: 1um. 